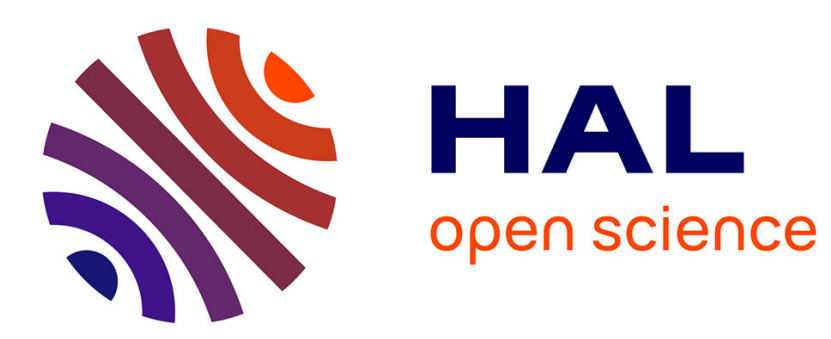

\title{
Slow modes in semi-dilute solutions of polynucleotides
}

P. Mathiez, G. Weisbuch, C. Mouttet

\section{To cite this version:}

P. Mathiez, G. Weisbuch, C. Mouttet. Slow modes in semi-dilute solutions of polynucleotides. Journal de Physique Lettres, 1978, 39 (9), pp.139-141. 10.1051/jphyslet:01978003909013900 . jpa-00231462

\section{HAL Id: jpa-00231462 https://hal.science/jpa-00231462}

Submitted on 1 Jan 1978

HAL is a multi-disciplinary open access archive for the deposit and dissemination of scientific research documents, whether they are published or not. The documents may come from teaching and research institutions in France or abroad, or from public or private research centers.
L'archive ouverte pluridisciplinaire HAL, est destinée au dépôt et à la diffusion de documents scientifiques de niveau recherche, publiés ou non, émanant des établissements d'enseignement et de recherche français ou étrangers, des laboratoires publics ou privés. 


\title{
SLOW MODES IN SEMI-DILUTE SOLUTIONS OF POLYNUCLEOTIDES
}

\author{
P. MATHIEZ, G. WEISBUCH and C. MOUTTET \\ Département de Physique, U.E.R. Scientifique de Luminy, \\ Université Aix-Marseille II, 13288 Marseille Cedex 2, France
}

(Reçu le 12 décembre 1977, révisé le 16 février 1978, accepté le 16 mars 1978)

\begin{abstract}
Résumé. - Nos études expérimentales de diffusion quasi élastique de la lumière par des solutions semi-diluées d'acide polyadénilique mettent en évidençe l'existence simultanée de modes rapides semblables à ceux observés avec des chaînes flexibles, et de modes lents dont l'existence semble due à la dynamique des chaînes de polynucléotides. La variation des coefficients de diffusion en fonction de la concentration donne quelques indications sur l'origine de ces modes.
\end{abstract}

Abstract. - Our experimental studies of quasi-elastic light scattering by semi-dilute solutions of polyadenilic acid show the simultaneous existence of rapid modes similar to those observed with flexible chains, and of slow modes the existence of which is due to polynucleotide chain dynamics. The concentration dependence of diffusion coefficients gives some indications about the origin of these modes.

Recently, a number of papers have been devoted to the study, by light scattering, of semi-dilute solutions of flexible polymers from both theoretical [1] and experimental points of view $[2,3]$. There exists a rather good agreement between theory and experiment on this type of materials.

The situation is much less favourable as far as rigid polymers like DNA are concerned [4-10]. Experimenters are aware of the fact that interactions between chains might be responsible for some of the surprising behaviour of the scattered light, but one actually misses a theoretical corpus that would allow a clear understanding of the experimental results. In this paper, we report our study of slow modes as a function of polymer concentration in polyadenilic acid semi-dilute solution.

Samples preparation and characterization. - Polyadenilic acid is a ribonucleic acid in which the only base is adenine. We start from commercial samples (Miles laboratories) prepared by synthesis. Their sedimentation coefficient is $S_{20, \mathrm{w}}=15.4$ in $0.1 \mathrm{M} \mathrm{NaCl}, 0.01 \mathrm{M}$ sodium phosphate buffer $\mathrm{pH}$ 7.0. All preparations and experiments are made under sterile conditions. The sample is fractionated by reversible phase separation in saline water solution according to a technique described by Eisenberg and Felsenfeld [11]. Special care is taken to make sure that no dust is present that would possibly alter our results. Since all solvents are filtered on $0.022 \mu$ Millipore before use, dust could then only come from the lyophilised poly $\mathrm{A}$ itself. In the process of phase separation, dust is very efficiently removed by the high salinity of the solution $(1.2 \mathrm{M} \mathrm{NaCl})$ and by the fact that we work only with intermediate fractions. Ultracentrifugation analysis does not give any evidence of inhomogeneities (like dust, aggregates, etc...).

The molecular mass is of the order of $3.5 \times 10^{6}$. Light scattering experiments are done in $0.1 \mathrm{M} \mathrm{NaCl}$, $0.01 \mathrm{M}$ sodium cacodylate buffer $\mathrm{pH}$ 7.4. The radius of gyration $R_{\mathrm{G}}$, estimated by elastic light scattering, is $2500 \AA$.

Experimental set-up. - The experimental set-up is standard. We use an argon laser (model 165 Spectra Physics). The incident light (wavelength $4880 \AA$, power from 10 to $800 \mathrm{~mW}$ ) is focused on the poly A solution. Scattered light, at an angle $\theta$ from the incident light, is focused on the photocathode of a FW 130 ITT photomultiplier. The amplifiers, the discriminator and the 48 channel digital autocorrelator are from «Precision Devices Malvern model K 7023 ». The detection is homodyne.

Data analysis. - The important fact in the analysis of our data is that the autocorrelation function of the scattered light intensity $A(t)$ involves two relaxation times. It cannot be fitted either by a single exponential or by De Gennes, Dubois-Violette function [12]. We then have chosen to fit our data with two exponentials

$$
A(t)=A+\left(B \mathrm{e}^{-t / \tau_{1}}+C \mathrm{e}^{-t / \tau_{r}}\right)^{2} .
$$


Since $\tau_{1}$ and $\tau_{\mathrm{r}}$ are very different, we first take a long sampling time to get rid of the rapid exponential $\tau_{\mathrm{r}}$. Leaving the first channels, for which the contribution of the rapid exponential cannot be neglected, we fit with one exponential plus a floating base line. Then, knowing $\tau_{1}$, we are able to make a three parameter fit of $|A(t)-A|^{1 / 2}$ where $A$ is measured from the monitor channels.

The variation of both relaxation times with the scattering wavevector are displayed on figure 1 . It can be seen that they differ by at least one order of magnitude. The ratio of their amplitudes is displayed on figure 2 .

Let us first consider the behaviour of the rapid relaxation time :

- For small wavevectors $\tau_{\mathrm{r}}$ varies as $q^{-2}$.

- For large wavevectors $\tau_{\mathrm{r}}$ varies as $q^{-2.6}$.

- The magnitude of the wavevector corresponding to the transition between these two behaviours decreases with concentration.

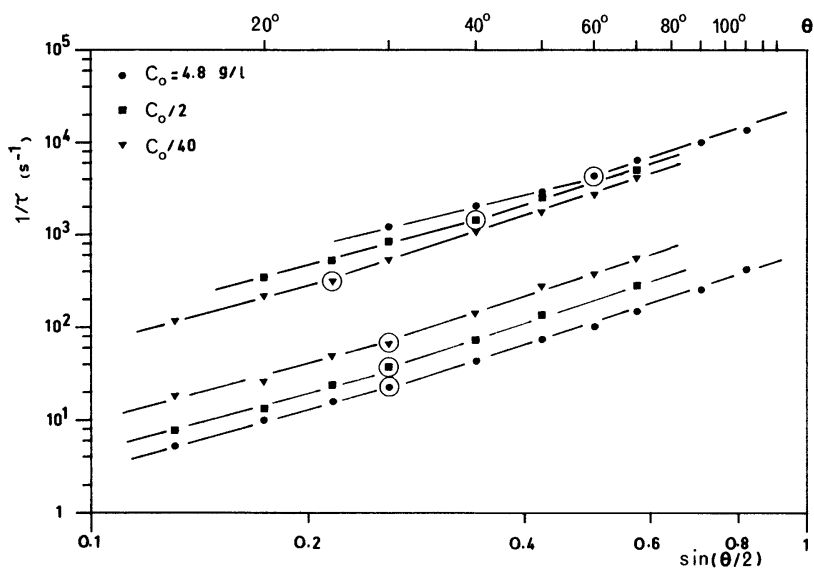

FIG. 1. - Log-Log plot of the inverse relaxation time as a function of the scattering wavevector. The different curves correspond to different concentrations $\left(C_{0}=0.013\right.$ moles per litre $\left.=4.8 \mathrm{~g} / \mathrm{l}\right)$. Both rapid and slow relaxation times are figured. The circles correspond to transitions between different physical regimes (from semi-dilute to quasi isolated in the upper curves) characterized by different slopes (from 2 to 2.6 )

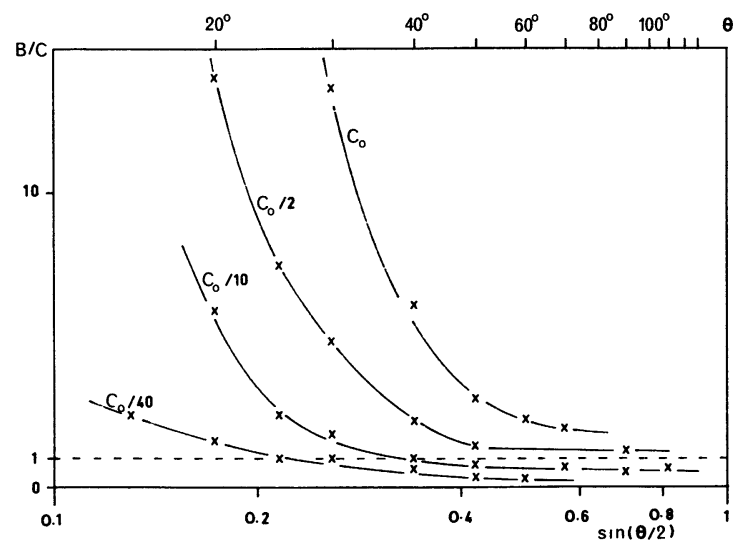

Fig. 2. - Plot of the relative amplitudes of rapid and slow modes as a function of the scattering wavevector for different concentrations.
This behaviour is very reminiscent of that of flexible polymers. Small wavevectors would correspond to collective excitations as described by $\mathrm{De}$ Gennes [1] and large wavevectors would correspond to modes of quasi isolated chains. The transition should occur for wavevectors of the order of $q \xi$ where $\xi$ is an entanglement characteristic length [13]. At present, we have no reliable model to determine the possible scaling laws relating $\xi$ to concentration for rigid polymers, but $\cdot$ it is certain that $\xi$ decreases with concentration.

On the other hand, the very existence of the slower mode appears as a characteristic feature of rigid chains. Large concentrations and small scattering wavevectors yield a relatively larger amplitude to the slow mode, which nearly disappears for small concentrations and large wavevectors (Fig. 2).

Figure 3 shows the variation of a diffusion coefficient defined by

$$
D=\left(\tau q^{2}\right)^{-1}
$$

for both modes as a function of concentration ( $D$ is calculated in the small wavevector region where the relaxation time obeys $\tau q^{2}=$ constant).

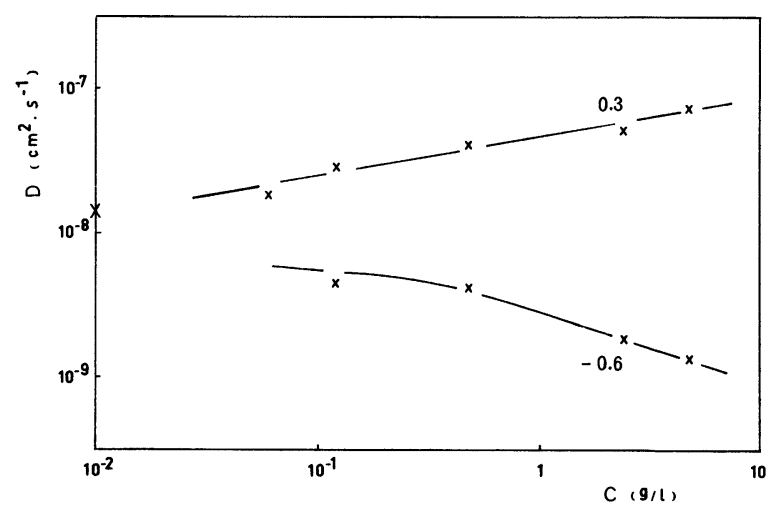

Fig. 3. - Log-Log plot of the diffusion constant of the rapid and slow modes as a function of concentration. The numbers are an indication of the asymptotic slopes of these curves. The cross on the vertical axis corresponds to an estimate from ultracentrifugation of the diffusion constant of an isolated chain of polyadenilic acid.

The rapid diffusion coefficient increases with concentration which is an indication of its collective nature. The slow diffusion coefficient decreases with concentration, possibly suggesting motion of individual chains hindered by the vicinity of neighbouring chains.

Since the diffusion coefficient of the slow mode seems to level off at low concentration, we can get an estimate of the hydrodynamical radius of the scatterers by using Stokes formula. We find $1 \mu$ which would imply that the slow mode is due to coherent motion of large parts of the chain, or possibly of the whole chain.

At this point, we should be able to propose a tentative explanation of the difference of behaviour between 
rigid and flexible chains : for flexible chains, entanglement points restrict the possibility of coherent movement of parts of the chains larger than $\xi$. This is not the case for rigid chains because their persistence length can be larger than $\xi$. Thus rigid chains can give rise to coherent motion of the whole chain even if it is entangled and this could be the origin of the slow modes.

In summary, the autocorrelation function of the scattered light by semi-dilute solutions of polynucleo- tides like poly $\mathrm{A}$ is characterized by two relaxation times. The rapid exponential behaves in a manner very similar to what is observed with flexible chains. On the other hand, the large persistence length of polynucleotide gives rise to the simultaneous existence of a slow mode, probably involving hindered motion of a chain relative to its neighbours.

Acknowledgments. - We thank Dr. F. Salvan for helpful discussions.

\section{References}

[1] De Gennes, P. G., Macromolecules 9 (1976) 587.

[2] Adam, M., Delsanti, M. and Jannink, G., J. Physique Lett. 37 (1976) 53.

[3] Adam, M. and Delsant, M., to appear in Macromolecules.

[4] Dubin, S. B., Lunacek, J. H. and BenedeK, G. B., Proc. Nat. Acad. Sci. U.S. 57 (1967) 1164

[5] SchmidT, R. L., Biopolymers 12 (1973) 1427.

[6] Schmitz, K. S. and Schun, J. M., Biopolymers 12 (1973) 1543.

[7] Schmitz, K. S. and Pecora, P., Biopolymers 14 (1975) 521.

[8] Jolly, D. and EISENBERG, H., Biopolymers 15 (1976) 61.

[9] Chen, F. C., Yeh, A. and Chu, B., J. Chem. Phys. 66 (1977) 1290.
[10] Schmidt, R. L., Boyle, J. A. and Mayo, J. A., Biopolymers 16 (1977) 317.

[11] Eisenberg, H. and Felsenfeld, G., J. Mol. Biol. 30 (1967) 17.

[12] De Gennes, P. G. and Dubois-Violette, E., Physics 3 (1967) 181.

[13] More precisely, as in the case of flexible chains, $\xi$ can be defined as the distance such that the pair correlation function of monomers of the same chain equals the square of the mean density of monomers. But, for rigid chains, $\xi$ is no more a screening length for interactions along the chain. 